\title{
Evaluation of The L2 Spinal Nerve Root Infiltration as A Diagnostic Tool for Discogenic low Back Pain
}

\author{
Robert Mendez, DO , Steven Bailey, MD, Gregory Paine, MD, Michael Mazzilli, MD, Eric Stedje-Larsen, MD, \\ Ben Nance, MD, and Kieth Dietrick, MD
}

\begin{abstract}
Background: To assess whether unilateral L2 infiltration with local anesthetic can be used to identify patients who will have negative discograms and thus eliminate the need for the discogram. Discogenic low-back pain is considered to have afferent pathways in the sinuvertebral nerves, mainly originating from the ventral rami of the spinal nerves. There is evidence that pain arising from the lower lumbar intervertebral discs may be transmitted through the sympathetic afferent fibers contained in the L2 spinal nerve root. Provocative discography, within the context of other clinical data, is the current "gold standard" by which to diagnose discogenic low-back pain, but a far more invasive procedure than $\mathrm{L} 2$ infiltration.
\end{abstract}

Objective: To evaluate the correlation between unilateral second lumbar (L2) spinal nerve root infiltration with local anesthetic and provocative discography in patients with chronic low back pain.

Study Design: A prospective, observational study.

Methods: All patients scheduled for discography were asked to participate in having local anesthetic infiltration of the L2 spinal nerve root at least two weeks prior to discography, until forty subjects were enrolled. Discography was performed after the patient's pain level returned to baseline.

Results: Local anesthetic infiltration of the L2 spinal nerve root was predictive of provocative discography results in only
$46.5 \%$ of the subjects $(26 \%$ true positives, and $20.5 \%$ true negatives). In $53.5 \%$ of the subjects, L2 infiltration was not predictive of discography results $(20.5 \%$ false positives, and $33 \%$ false negatives).

Conclusions: The results showed that unilateral L2 infiltration is not predictive of discogenic low-back pain when compared to discography, the current "gold-standard" for diagnosis.

Keywords: L2 spinal nerve infiltration, discogenic low back pain, provocative discography, sympathetic afferent pathways, selective nerve root block, sinuvertebral nerve
The lifetime incidence of low back pain in the general population is estimated to be $60 \%$ to $80 \%$. At a cost of $\$ 24$ billion per year, the medical treatment of low back pain is the leading compensable cost of injury in the workplace (1). Research suggests that degenerating intervertebral discs are the primary source of low back pain. Conservative management, to include medication, physical therapy, rest, modalities, and patient education, is ineffective in cases of chronic low back pain

From Department of Anesthesia and Pain Medicine Clinic, Naval Medical Center, Portsmouth, Virginia. Address Correspondence: Michael Mazzilli, MD, 27 Effingham St., Portsmouth, VA 23708.

Disclaimer: The views expressed in this article are those of the authors and do not reflect the official policy or position of the Department of the Navy, Department of Defense, or the United States Government.

Conflict of Interest: None

Acknowledgement:

Manuscript received on 8/18/04

Revision submitted on $12 / 13 / 04$

Accepted for publication on $12 / 13 / 04$

Additional Disclosures are listed with author affiliation details.
(2). Discogenic low back pain is frequently managed with surgical fusion or less invasive procedures such as intradiscal electrothermal annuloplasty (IDET).

The current "gold standard" for the diagnosis and identification of the affected disc level(s) is provocative discography $(3,4)$. Discography utilizes several objective measures in the evaluation of a disc, including volumetric, manometric, and radiographic data. The crux of the test rests in the patient's report of pain location and intensity. This subjective component of the test is adversely affected in patients with abnormal psychological profiles, resulting in a greater false positive rate $(5,6)$. Additionally, provocative discography is superior to magnetic resonance imaging (MRI) in the evaluation of discogenic pain (7-14).

Discogenic low-back pain is considered to have afferent pathways in the sinuvertebral nerves, which on anatomic dissection merge with the ventral rami of the spinal nerves. However, many patients with disc herniation complain of sciatica without low-back pain (15). This sug- gests that the spinal nerve roots are being compressed proximal to the branching point of the sinuvertebral nerves and that the afferent pathways for discogenic lowback pain are not in the spinal nerves at the same level. There is evidence that pain arising from the lower lumbar intervertebral discs is transmitted non-segmentally via the paravertebral sympathetic chain, and then through the visceral sympathetic afferent fibers contained in the L2 spinal nerve root (Fig. 1) (16-17). Thus L2 infiltration is a possible diagnostic tool and could be used for the conservative treatment of discogenic low-back pain.

Nakamura et al (15) performed unilateral L2 infiltration in 33 patients that met their criteria for discogenic low-back pain based on clinical diagnosis by physical exam, plain films, and MRI. They observed pain relief in all 33 patients for an average of 20.7 days ( 1.5 hours to 100 days).

The purpose of this study was to assess whether L2 local anesthetic nerve root infiltration can be used to identify patients who will have negative disco- 


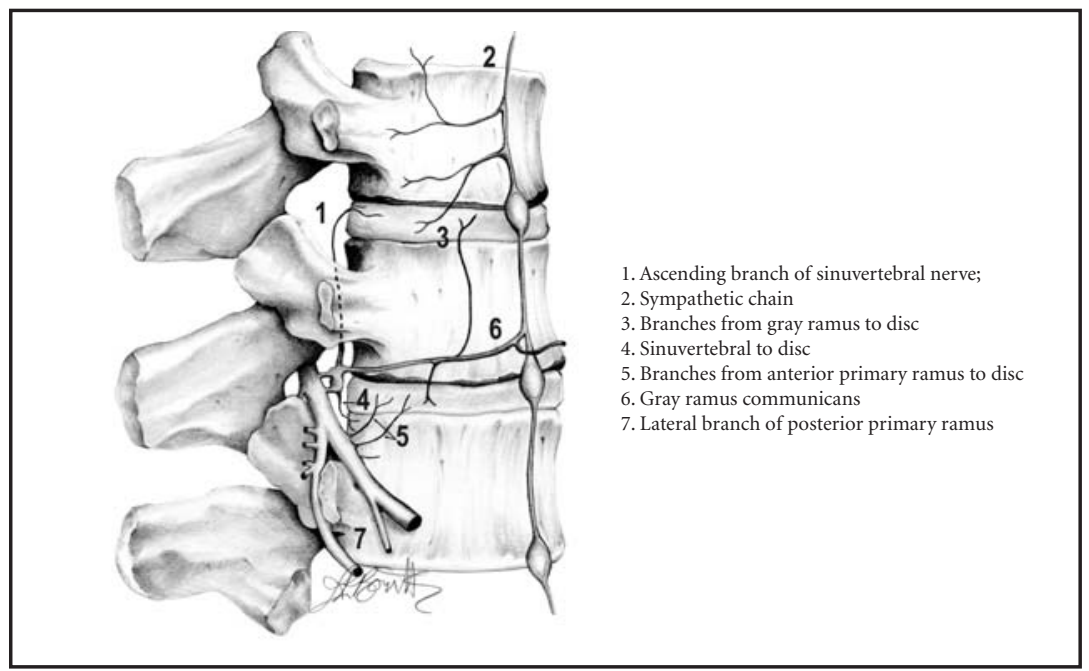

Fig 1. L2 spinal nerve root

grams secondary to having a non-discogenic etiology of their low back pain. This would eliminate unnecessary diagnostic provocative discography in those patients, thereby reducing the risks and procedural related pain. The principle risk inherent with performing discography is the exacerbation of existing pain. Other risks of discography include bleeding, infection, discitis, damage to surrounding structures, and allergic reaction to agents used in performing the procedure. Discography would still be necessary in those patients with a pain relieving response to L2 nerve block, in order to identify and better characterize the involved disc level(s).

\section{Methods}

The study was undertaken in the Pain Medicine Clinic of the Naval Medical Center Portsmouth, VA. The protocol was approved by the Institutional Review Board at Naval Medical Center, Portsmouth, VA. The design consisted of a prospective, observational evaluation.

\section{Informed Consent}

All patients were provided with the approved protocol and informed consent document, approved by the Institutional Review Board for this study. The informed consent document described the details of the evaluation.

\section{Inclusion and Exclusion Criteria}

All the patients who participated in the study were identified from the referral pool of the patients being referred to the Pain Medicine Clinic, Naval Medical Center. All patients had received diagnos- medical evaluation, information on previous treatments, and pain assessment.

\section{Study Design and Investigation}

A single group of 40 patients received both L2 local anesthetic nerve root infiltration and discography. Discography was performed at a minimum of 2 weeks after L2 nerve root infiltration, but more importantly, only after the patient's pain returned to baseline, which in no subject was greater than 8-weeks. Patients maintained a pain diary recording their pain by the Verbal Numeric Score (VNS) at 48 hours after L2 block and then weekly. Pain Clinic nurses called the patients weekly to ascertain each patient's pain level and record the score.

The L2 nerve root infiltration was carried out on the patient's predominantly painful side in the prone position. The skin was anesthetized with $1 \%$ Lidocaine (Xylocaine-MPF 1\% AstraZeneca, Wilmington, DE). A 25g Quinke Spinal needle (Kimberly-Clark spinal tray, Roswell, GA) was advanced to the L2 foramen under fluoroscopic guidance (OEC Series 9600, GE Medical Systems, Raleigh, NC). Proper needle placement was confirmed in both anteroposterior and lateral views before and after injecting $1 \mathrm{~mL}$ of contrast (Omnipaque 300, Amersham, Princeton, NJ) to outline the L2 nerve root. The nerve root was then anesthetized by injecting $1.5 \mathrm{~mL}$ of $2 \%$ Lidocaine (Lidocaine-PF 2\%, Abbott Labs, Chicago, IL). Pain relief was assessed by the VNS. The patient' pain score was recorded immediately preceding L2 infiltration and again 20 minutes after the procedure. Pain relief was considered positive if there was at least a $50 \%$ decrease in VNS, otherwise negative.

The provocative discography procedure was initiated opposite the patient's predominantly painful side in the prone position, in accordance with technique described elsewhere $(4,18)$. The skin was prepped with Betadine solution and drapes applied utilizing sterile technique. Sedation was performed with aliquots of intravenous Propofol (Diprivan $1 \%$, AstraZeneca, Wilmington, DE). Skin was anesthetized with $1 \%$ Lidocaine. Under fluoroscopic guidance, 25g, 6" Quinke spinal needles were placed through 20g, 3.5" introducer needles (Nerve Root Block Kit, Kimberly-Clark, Roswell, GA) into the disc spaces that had corresponding degenerative changes on MRI. Once 
Table 1. Demographic characteristics and L2 infiltration versus discography

\begin{tabular}{|c|c|c|c|}
\hline \multirow{2}{*}{\multicolumn{2}{|c|}{ Gender }} & Male & $85 \%(34)$ \\
\hline & & Female & $15 \%(6)$ \\
\hline \multirow{3}{*}{\multicolumn{2}{|c|}{ Race }} & African American & $22 \%(9)$ \\
\hline & & Caucasian & $73 \%(29)$ \\
\hline & & Hispanic & $5 \%(2)$ \\
\hline \multicolumn{2}{|l|}{ Age } & Mean \pm SD & $38 \pm 8.7$ \\
\hline \multirow{2}{*}{ VAS pain score } & Baseline & Mean \pm SD & $5.7 \pm 1.73$ \\
\hline & 20 Minutes after L2 & Mean \pm SD & $3.7^{\star} \pm 1.93$ \\
\hline \multirow{2}{*}{\multicolumn{2}{|c|}{ L2 infiltration }} & Positive & $47 \%(29)$ \\
\hline & & Negative & $53 \%(21)$ \\
\hline \multirow{2}{*}{\multicolumn{2}{|c|}{ Discogenic }} & Positive & $59 \%(23)$ \\
\hline & & Negative & $41 \%(16)$ \\
\hline \multirow{4}{*}{\multicolumn{2}{|c|}{ Number of levels positive }} & Zero & $41 \%(16)$ \\
\hline & & One & $33 \%(13)$ \\
\hline & & Two & $23 \%(9)$ \\
\hline & & Three & $3 \%(1)$ \\
\hline
\end{tabular}

the patient was clearly no longer sedated, provocative discography was commenced by slowly injecting up to $1.5 \mathrm{~mL}$ of contrast (Omnipaque 300) into the disc spaces under fluoroscopy, utilizing a $20 \mathrm{cc}$ syringe with threaded plunger and manometric capability (Universal Fluid Dispensing Syringe, Merit Medical Systems, South Jordan, UT).

Each disc was interrogated sequentially by a principle investigator, with notation made of 1) opening pressure, in pounds per square inch, 2) whether or not pain was produced, 3 ) pressure (over opening) which caused pain, 4) concordance of the pain compared to the patient's typical pain, 5) severity of pain produced. The test was considered negative if the patient's typical back pain was not reproduced, and positive if all of the patient's pain, or a component of their pain in cases of multilevel involvement, was reproduced with any amount of contrast less than or equal to $1.5 \mathrm{~mL}$ and at a change in pressure of less than 50 pounds per square inch over the opening pressure,

Table 2. The predictive value of L2 infiltration as compared to provocative discography in 39 patients

\begin{tabular}{|c|c|}
\hline True Positive & True Negative \\
(Positive L2/Positive Disco) & (Negative L2/Negative Disco) \\
$25.6 \%(10)$ & $20.5 \%(8)$ \\
\hline False Positive & False Negative \\
(Positive L2/Negative Disco) & (Negative L2/Positive Disco) \\
$20.5 \%(8)$ & $33.3 \%(13)$ \\
\hline
\end{tabular}

neous with regard to age and co- morbidities. The patient population was, however, predominantly male ( 34 of 40 ), reflecting the predominantly male population in the Armed Services (Table 1). One patient withdrew from the study after undergoing L2 infiltration, but before discography, when he moved out of the area. There were no complications of either provocative discography or L2 infiltration. Measured outcomes of the L2 spinal nerve root infiltration and subsequent provocative discography are displayed by subject number in Table 1. Chi square analysis, as depicted in Table 2, was essentially random, and thereby demonstrates no correlation.

\section{DisCussion}

In contrast to the results of Nakamura et al (15) the results of this study do not support the effectiveness of unilateral L2 infiltration as a therapeutic modality in the relief of discogenic low-back pain. Nor do the results support the use of unilateral L2 infiltration as a diagnostic tool in the evaluation of discogenic lowback pain.

Ohtori et al (19) published an animal study a few years after the Nakamura et al study, in the same lab, which may help explain our study's results. They found that the posterior portion of the lumbar discs in rats were innervated by two distinct pathways: segmentally by the sinuvertebral nerves, and by non-segmental nerve fibers through the paravertebral sympathetic trunks. High variability in the anatomic innervation of the human disc may explain the variability of response to unilateral L2 nerve block when discogenic pain is present as proven by discography.

Of note, the current study took over two and a half years to collect 40 patients. During that time, there were seven different investigators at different levels of experience, some fellows in training, some staff pain physicians, performing the procedures and collecting the data. That may have allowed for some variation in technique and interpretation of clinical results.

The current study establishes the need for further research to determine more precisely the pathophysiology of discogenic low-back pain, as well as the continued need to develop conservative and cost effective means of diagnosing and treating discogenic low back pain. The authors are considering repeating the 
study employing bilateral L2 infiltration, rather than only unilateral infiltration. Many patients report bilateral low-back pain. Additionally, there is the possibility that there may be some right-left crossover in the innervation of the posterior lumbar disc.

\section{ConcLusion}

Unilateral L2 infiltration is not predictive of discogenic low-back pain when compared to discography, the current "gold-standard" for diagnosis. Whether this same conclusion would hold true utilizing bilateral L2 segmental nerve block is unknown.

\section{ACKNOWLEDGEMENTS}

William Douglas, RN and Carolyn Guenveur, RN for their invaluable assistance in the safe performance of the procedures carried out, and in data acquisition.

Thomas Rieg, PhD for his extremely helpful guidance in the statistical analysis of this project. Ms. Jean Bonnette for her invaluable assistance as a medical illustrator.

The authors also wish to thank editors of Pain Physician for peer review and constructive criticism, which ultimately improved the quality and understanding of the manuscript.

Federal Employment Disclosure: All the above listed authors were employees of the Federal Government at the time the research was conducted, specifically the Naval Branch of the Department of Defense. This work was prepared as part of the authors' official duties. Title 17 U.S.C. 105 provides that "Copyright protection under this title is not available for any work of the United States Government." Title 17 U.S.C. 101 defines a United States Government work as a work prepared by military service members as part of their official duties.

Sponsor: The Chief, Navy Bureau of Medicine and Surgery, Washington, DC, Clinical Investigation Program sponsored this study (CIP\#P02-0004). There was no other financial involvement with any organization or entity.

\section{Author Affiliation}

\section{Robert Mendez, DO}

Director

Pain Medicine Clinic

Naval Medical Center

27 Effingham St.

Portsmouth, VA 23708

\section{Steven Bailey, MD}

Pain Medicine Clinic Naval Medical Center 27 Effingham St.

Portsmouth, VA 23708

Gregory Paine, MD

Pain Medicine Clinic

Naval Medical Center

27 Effingham St.

Portsmouth, VA 23708

\section{Michael Mazzilli, MD}

Fellow, Pain Medicine Clinic

Naval Medical Center

27 Effingham St.

Portsmouth, VA 23708

E-mail: MAMazzilli@mar.med.navy.mi

Eric Stedje-Larsen, MD

Pain Medicine Clinic

Naval Medical Center

27 Effingham St.

Portsmouth, VA 23708

\section{Ben Nance, MD}

Department of Anesthesia

Pain Medicine Clinic

Naval Medical Center

27 Effingham St.

Portsmouth, VA 23708

\section{Kieth Dietrick, MD}

Staff, Pain Medicine Clinic

Naval Medical Center

27 Effingham St.

Portsmouth, VA 23708

\section{REFERENCES}

1. Fischgrund JS, Montgomery DM. Diagnosis and treatment of discogenic low back pain. Orthopaedic Review 1993; 22:311318.

2. Kuslich SD, Ulstrom CL, Griffith SL, Ahern JW, Dowdle JD. The Bagby and Kuslich method of lumbar interbody fusion. History, techniques, and 2-year follow-up results of a United States prospective, multicenter trial. Spine 1998; 23:1267-1279.

3. Donelson R, Aprill C, Medcalf R, Grant W. A prospective study of centralization of lum- bar and referred pain. A predictor of symptomatic discs and annular competence. Spine 1997; 22:1115-1122.

4. Anderson SR, Flanagan B. Discography. Curr Rev Pain 2000; 4:345-352.

5. Carragee EJ, Chen Y, Tanner CM, Truong T, Lau E, Brito JL. Provocative discography in patients after limited lumbar discectomy: A controlled, randomized study of pain response in symptomatic and asymptomatic subjects. Spine 2000; 25:3065-3071.

6. Carragee EJ, Tanner CM, Khurana S, Hayward C, Welsh J, Date E, Truong T, Rossi M, Hagle C. The rates of false-positive lumbar discography in select patients without low back symptoms. Spine 2000; 26:994996.

7. Buirski G, Silberstein M. The symptomatic lumbar disc in patients with low-back pain. Magnetic resonance imaging appearances in both a symptomatic and control population. Spine 1993; 18:1808-1811.

8. Ricketson R, Simmons JW, Hauser BO. The prolapsed intervertebral disc. The high-intensity zone with discography correlation. Spine 1996; 21:2758-2762.

9. Braithwaite I, White J, Saifuddin A, Renton P, Taylor BA. Vertebral end-plate (Modic) changes on lumbar spine MRI: correlation with pain reproduction at lumbar discography. Eur Spine J 1998; 7:363-368.

10. Carragee EJ, Paragioudakis SJ, Khurana S. Lumbar high-intensity zone and discography in subjects without low back problems. Spine 2000; 25: 2987-2992.

11. Borenstein DG, O'Mara JW Jr, Boden SD, Lauerman WC, Jacobson A, Platenberg C, Schellinger D, Wiesel SW.. The value of magnetic resonance imaging of the lumbar spine to predict low-back pain in asymptomatic subjects: A seven-year followup study. J Bone Joint Surg Am 2001; 83A: 1306-1311.

12. Fukuda K, Kawakami G. Proper use of MR imaging for evaluation of low back pain (radiologist's view). Semin Musculoskelet Radiol 2001; 5:133-136.

13. Sandhu HS, Sanchez-Caso LP, Parvataneni HK, Cammisa FP Jr, Girardi FP, Ghelman B. Association between findings of provocative discography and vertebral endplate signal changes as seen on MRI. J Spinal Disord 2000; 13:438-443.

14. Boos N, Semmer N, Elfering A, Schade V, Gal I, Zanetti M, Kissling R, Buchegger N, Hodler J, Main CJ.. Natural history of individuals with asymptomatic disc abnormalities in magnetic resonance imaging: predictors of low back pain-related medical consultation and work incapacity. Spine 2000; 25:1484-1492.

15. Nakamura SI, Takahashi K, Takahashi Y, Yamagata M, Moriya H. The afferent pathways of discogenic low-back pain. Evaluation of L2 spinal nerve infiltration. J Bone Joint Surg Br 1996; 78:606-612.

16. Suseki K, Takahashi Y, Takahashi K, Chiba T, Yamagata M, Moriya H. Sensory nerve fibers from lumbar intervertebral discs 
pass through rami communicantes. A possible pathway for discogenic low back pain. J Bone Joint Surg Br 1998; 80:737742.

17. Nakamura S, Takahashi K, Takahashi Y, Morinaga T, Shimada Y, Moriya H. Origin of nerves supplying the posterior por- tion of lumbar intervertebral discs in rats. Spine 1996; 21:917-924.

18. Sachs BL, Spivey MA, Vanharanta H, Guyer RD, Rashbaum RF, Hochschuler SH, Scala AD. Techniques for lumbar discography and computed tomography/discography in clinical practice. Orthop Rev 1990; 29:
775-778.

19. Ohtori S, Takahashi Y, Takahashi K, Yamagata M, Chiba T, Tanaka K, Hirayama J, Moriya $\mathrm{H}$. sensory innervation of the dorsal portion of the lumbar intervertebral disc in rats. Spine 1999; 24:2295-2299. 
